DOI: 10.17234/SRAZ.65.57

\title{
Lo strano caso de Il Diritto Croato di Pola
}

\author{
Fabrizio Fioretti \\ Università Juraj Dobrila di Pola \\ ffiore@unipu.hr
}

Il Diritto Croato fu un settimanale croato in lingua italiana pubblicato a Pola a partire dal 1888. Data la situazione sociale dell'Istria di fine Ottocento, che vide Italiani e Croati scontrarsi sia sul piano politico e sia su quello culturale, le riviste divennero veri centri propulsori della storia e della letteratura. Onde poter arginare lo strapotere dei giornali italiani, tra le pagine de Il Diritto Croato trovarono così spazio centinaia di opere letterarie croate tradotte da personaggi in gran parte celati dietro pseudonimi. A margine di queste considerazioni, nel saggio si riscontra come la traduzione nell'Istria di fine Ottocento debba essere considerata un mezzo adottato nella diatriba culturale tra Italiani e Croati per dimostrare la grandezza della letteratura dell'Est europeo a discapito di quella occidentale e avvicinare così la popolazione dell'Istria alla causa croata.

Parole chiave: Il Diritto Croato, Ante Jakić, Istria, Ottocento, periodici italiani dell'Istria.

Anti-italiano, strenuo difensore dei diritti del popolo croato e, in generale, di tutti i popoli dell'Est Europa, Il Diritto Croato rappresenta uno dei casi più singolari dell'intera e già di per sé intricatissima storia giornalistica e politica dell'Istria dell'Ottocento (Cella 1956). Pubblicato a Pola a partire dal 3 ottobre 1888, questo settimanale croato in lingua italiana vede la luce grazie a tal Ante (o Antun o Antonio) Jakić che, solo alcuni anni addietro, dalla nativa Podgora in Dalmazia, si era trasferito nella città istriana in veste di cappellano di marina (Bertoša/Matijašić 2005: 179). L'avventura de Il Diritto Croato si conclude nel corso del suo sesto anno col numero 25 del 21 marzo 1894 quando, a causa della vita sregolata e vezzosa del suo fondatore che lo porterà a contrarre numerosi debiti, lo Jakić passa a Trieste dove continuerà la sua avventura giornalistica prima con Il Pensiero Slavo, poi con La Pensée Slave ed infine con il settimanale Slavenska Misao. ${ }^{1}$

Senza doverci addentrare in ulteriori disquisizioni di carattere tecnico, per altro già affastellate in un articolo precedente (Fioretti 2019: 113-126), onde poter

1 Si desume questo fatto da una nota apparsa su Il Diritto Croato nella quale è lo stesso Jakić a dichiarare di aver contratto un debito pari a 3000 fiorini. A tal proposito si veda il numero del 7 marzo 1894, VI, 23. 
procedere oltre è necessario tuttavia sintetizzare alcuni punti senza i quali la comprensione del tema che si sta per affrontare risulterebbe alquanto lacunosa. Innanzitutto vi è da dire che questo settimanale ancora oggi risulta parecchio misterioso. Ad esempio sul suo fondatore, che una volta a Pola abbandonerà ben presto l'abito talare, non sappiamo molto e soprattutto non è dato sapere chi finanziasse le sue imprese giornalistiche. Aleggiano molte strane dicerie, già avanzate nel corso dell'Ottocento, una delle quali lo vorrebbe collaboratore dei servizi segreti russi se non addirittura una spia dello Zar Nicola II. ${ }^{2}$ Inoltre nulla si sa sul suo destino dopo la chiusura della Slavenska Misao nel luglio 1909 nemmeno l'anno della sua dipartita. Jakić a parte, un altro punto da porre in rilievo riguarda il fatto che non è stato ancora concretamente studiato il ruolo che questo foglio ha occupato all'interno del rinascimento nazionale croato in Istria nel corso del XIX secolo. Infatti, da un'attenta analisi degli studi sin qui condotti, appare incredibile il fatto che su Il Diritto Croato non vi sia che qualche citazione o qualche frettolosa e spesso errata conclusione (Fioretti 2019: 113-114).

Per quanto misteriosi e financo strani possano apparire alcuni aspetti di questo settimanale, quello che tra il 1888 e il 1894 esce ogni mercoledì dalla tipografia Seraschin di Pola non va assolutamente visto come una sorta di coincidenza. Il Diritto Croato, infatti, è figlio del suo tempo ossia di quella vera e propria battaglia politico-sociale, ma anche e soprattutto culturale tra Italiani e Croati avviata, com'è noto, con la rinascita nazionale croata di questa regione (Ivetic 2006: 461-530). Proprio per questo motivo, fin dal suo arrivo a Pola nel 1885 lo Jakić si rese conto che mentre l'intellighenzia italiana dell'Istria, sebbene non in maniera omogenea, aveva dato vita a una vera e propria macchina di propaganda politica con decine tra quotidiani, settimanali e mensili, senza parlare dell'incredibile impulso dato agli studi storici con i quali si voleva dimostrare la millenaria presenza degli Italiani in questa regione (Knez 2015), la parte croata aveva risposto, quasi timidamente da Trieste (poi però da Pola) con la sola Naša Sloga. Inoltre, e com'è lecito immaginare, tra quest'ultima e riviste quali, ad esempio, L'Istria di Parenzo era in atto una vera e propria battaglia basata su luoghi comuni che spesso e volentieri sfociava in offese anche razziste (Damiani/Fioretti 2017: 57-58).

A margine della condizione di svantaggio del giornalismo croato in questa regione, Jakić decide di inserirsi in questa annosa e complessa battaglia tra Italiani e Croati, ma non con un giornale in lingua croata, bensì con uno in lingua italiana cosa, per altro, nuova in Istria ma non nella sua Dalmazia visti i casi dell' Avvenire (1848-1849) di Ragusa e del Nazionale (1862-1871 diventato poi Narodni List) di Zara. Nacque così Il Diritto Croato un settimanale, in fondo, simile a molti altri dell'epoca, nel quale interventi politici e patriottici si alternavano alle rubriche culturali e letterarie. Proprio quest'ultimo dato non deve stupire: quella tra le due etnie più numerose della penisola istriana, infatti, fu una lotta che si giocherà soprattutto in ambito storico e culturale, tanto da sostenere che se non ci fosse

2 A tal proposito si desidera sottolineare che tali voci vennero avanzate soprattutto da giornali italiani ostili allo Jakić come, uno su tutti, L'Eco di Pola con il famoso articolo Lo storto di Pola (8 agosto 1891, VI, 289). 
stata questa, l'Istria - molto probabilmente - non avrebbe vissuto quello che è da ritenere il suo periodo letterario più florido. Più dei lunghi discorsi tra le mura dell'allora Parlamento Istriano, le intellighenzie della penisola videro proprio nello sviluppo della stampa periodica di quegli anni il mezzo ideale per allargare tra la popolazione un forte senso di appartenenza nazionale cosa, quest'ultima, possibile soprattutto attraverso la storia e la cultura. Saggi, analisi e recensioni letterarie, ma anche poesie o romanzi d'appendice rappresentavano veri e propri vettori patriottici in grado di inorgoglire il pubblico a cui erano destinati, in quanto dimostravano la grandezza di una nazione. In tal senso diventa ovvio perché allora tutti scrivessero e soprattutto perché l'Istria dell'Ottocento venne letteralmente inondata di cultura. Sia però chiaro che la questione, almeno in ambito italofono, è molto più complessa di quanto si potrebbe immaginare siccome se da un lato, forti del pensiero irredentista, intellettuali del calibro di Paolo Tedeschi spingeranno i lettori a dar vita a una letteratura Nuovo Romantica (o, se ci è concesso dire, moralistico-cattolico-patriottica) sul modello di Manzoni e De Amicis, altri, come lo zaratino Gerolamo Enrico Nani Mocenigo tenteranno di inorgoglire la popolazione istriana attraverso le grandi novità e le grandi rivoluzioni letterarie dell'Italia di allora, ispirandosi al Verismo (Damiani/Fioretti 2017: 14-73).

Interventi e digressioni politiche a parte, Il Diritto Croato vedrà la luce anche con lo scopo di limitare lo strapotere delle riviste italiane tentando di contrastare, per quanto possibile, l'imponente meccanismo di propaganda culturale dei giornali italiani. Detto in altre parole, chiunque decida di esaminare questo periodico deve farlo prendendo in considerazione anche la letteratura e del resto basta sfogliare alcuni numeri per rendersene conto. Lo Jakić e i suoi numerosi collaboratori, infatti, nel giro di qualche anno daranno vita a quello che già Arturo Cronia aveva segnalato come una «Nuova rassegna bibliografico-letteraria di Firenze» (1958: 571). Sulle pagine de Il Diritto Croato, per l'appunto, si possono leggere decine di lunghi e complessi studi su Petar Preradović, Andrija Kačić Miošić e Ivan Mažuranić, e soprattutto numerosissime traduzioni in italiano di poesie popolari, racconti vari e opere del livello di Propast Venecije [La caduta di Venezia] di August Šenoa, tutte racchiuse nell'importantissima rubrica Musa Slava. Già da questa brevissima descrizione diventa evidente che Il Diritto Croato era il più grande centro propulsore della cultura croata, e in generale di tutte le letterature slave, in questa regione e proprio in funzione di ciò oggi è da considerarsi come la più grande e la più completa antologia della letteratura croata (e non solo) dell'Ottocento; un'opera attuale, utile e, per tanto, decisamente futuribile. ${ }^{3}$

A margine di queste considerazioni, si fa largo una domanda ineludibile e che, per sommi capi, si tenterà di argomentare in questa sede ovvero che cosa si intende per traduzione in un contesto simile? Se partiamo dall'idea che tradurre

3 Come accennato, non solo vennero tradotte opere dal croato all'italiano ma anche moltissime poesie, racconti, canti popolari e saggi di autori russi, bulgari, sloveni, serbi e cechi. 
significhi «dire la stessa cosa in un'altra lingua» (Eco 2012: 9) o, detto altrimenti, un «[...] processo mediante il quale una lingua di partenza (LP) viene resa nella lingua di arrivo (LA) in modo che il significato superficiale delle due lingue sia più o meno simile» (Bassnett 2003: 14) allora anche il nostro periodico, come del resto gran parte dei testi tradotti, rientra perfettamente entro quest'ottica. La cosa però è ben più complessa di come appare e basta alzare un po' l'asticella o, per meglio dire, fare un passo in avanti tra gli Translation studies per rendersene conto. Riprendendo le parole di Gianfranco Folena che nel suo Volgarizzare e tradurre aveva accostato la traduzione alla tradizione, Siri Nergaard dichiara:

La traduzione è un luogo privilegiato per far incontrare lingue, letterature e culture; per unire il passato e il presente, il lontano e il vicino; per creare insomma quella che Goethe chiama una Weltliteratur (una letteratura mondiale) e infine per creare una tradizione (1993: 7).

Essendo di fronte a un periodico croato, ma in lingua italiana, c'è da chiedersi innanzitutto a chi era rivolto ovvero chi fossero i destinatari di tutta questa mole di letteratura croata in lingua italiana. Diciamo subito che non siamo del tutto d'accordo con le idee di Cella che sosteneva che questo era destinato ai Croati che non capivano il croato e agli Italiani che desideravano essere informati sul partito avversario (1956: 131-132). Già dalla lettura del Programma, visti i propositi di lottare per tutto il popolo croato, è evidente che a leggerlo dovevano essere anche i lettori croati che conoscevano sia l'una sia l'altra lingua; tuttavia i veri destinatari, almeno per quanto concerne il lato culturale, erano gli Italiani dell'Istria. Come dichiara lo Jakić:

[...] noi lotteremo da forti contro l'intolleranza ingenerosa, l'ignoranza imperdonabile, l'invidia bassa e l'odio non meritato, cui i Croati son fatti bersaglio in casa propria e fuori. [...] e per rendere facile tale opera, servendoci della lingua italiana, e illustrando le condizioni storiche civili e letterarie della nazione croata, porremo speciale cura a distruggere fra gli avversari, e più ancora fra tutti gli imparziali ed onesti, gli assurdi, enormi pregiudizi, sulla base dei quali con incredibile leggerezza o malafede i Croati sono giudicati da quelli che più potrebbero e dovrebbero conoscerli ed apprezzarli degnamente. ${ }^{4}$

Già dalla lettura di questo brano si evince quanto Il Diritto Croato sia lontano da concetti quali incontro culturale e tradizione e, del resto, lo è anche quasi tutta la letteratura istriana dell'epoca tanto da essere spesso definita un fenomeno più battagliero che meramente culturale (Maier 1996: 53). Con ciò si vuole insinuare che non sempre traduzione equivale a tradizione, ma che talvolta può significare anche competizione culturale. Come lo stesso Jakić ribadirà fin dal primo numero, non solo per lui la missione «dell'arte è nobile e alta», ma dando ampio risalto alla letteratura si fa un atto altamente patriottico e, aggiunge:

Con frequenti e variati saggi critici e con opportune traduzioni, noi porgeremo loro (agli Italiani $i^{5}$ ) occasione d'imparar a conoscere più davvicino

4 Il Nostro programma, in Il Diritto Croato, Pola, 3 ottobre 1888, I, 1.

5 La nota è nostra. 
le letterature slave, piene di giovanile freschezza, di sana energia e atte [...] a trasfondere un forte alito di vita nuova nell'esaurito spirito letterario delle nazioni occidentali. ${ }^{6}$

Quello che il Nostro cercava non era il dialogo tra letterature e non era nemmeno il desiderio di dare vita a una unione tra Est ed Ovest, come magari erroneamente si potrebbe pensare dal brano testé riportato, bensì era lo scontro letterario, era il vano tentativo di dimostrare la superiorità della letteratura croata e di tutta quella dell'Est europeo, su quella occidentale per, come detto, tentare di indebolire tra gli Italiani il loro orgoglio nazionale. In tal senso basterebbe citare le numerose diatribe sulla presunta nazionalità (croata o italiana) di Niccolò Tommaseo, ma ci piace, invece, riportare un altro esempio. In un trafiletto dal titolo Un giudizio del Carducci sulla lingua croata, Jakić riproduce un articolo pubblicato sul periodico Obzor di Zagabria nel quale Carducci, di fronte alla richiesta di tradurre alcune sue poesie, tesseva le lodi della lingua croata. Ecco che cosa il Nostro scrive agli Italiani d'Istria: «Che ne diranno ora quei degeneri figli del popolo italiano, trovantisi al di qua dell' Adria, i quali non amano nulla di meglio che di oltraggiare l'eroico popolo croato e d'irridere la sua dolce favella?».? Traduzione, insomma, che in un ambiente simile può essere considerata alla stregua di uno strumento più bellico che di pace.

Data la natura sintetica di questo saggio, che di fatto vuole suggerire l'idea che la traduzione non sempre sia da considerare come uno scambio culturale, vi è da dire che intorno al presente tema vertono una serie di quesiti tutt'altro che secondari e che sono legati alla figura del traduttore. Commentando Dopo Babele, Franco Buffoni dichiara che George Steiner aveva intuito come, ancor prima di essere un esercizio formale, la traduzione sia un'esperienza esistenziale (2007: 21). Partendo da questa posizione vi sarebbe da capire se effettivamente, nello specifico caso de Il Diritto Croato, il traduttore non sia solo qualcuno che fa un atto meccanico ovvero che riscrive in un'altra lingua un testo (Bassnett 2003: 15), ma sia piuttosto un traduttore militante ossia qualcuno che rivivendo l'atto creativo trasporta dal croato all'italiano non solo le parole ma la cultura, il patriottismo e tutti i sentimenti espressi nei testi; una figura che mai come in questo caso deve rendere ancor più enfatica una poesia o un racconto e che deve essere in grado di passare al lettore tutti i suoi sentimenti e soprattutto il suo amore per la patria tentando di avvicinarlo alla causa croata. Un quesito questo che richiede una lunga e complessa analisi anche perché se da un lato la maggior parte dei traduttori erano anonimi, dall'altro, i pochi che hanno firmato le traduzioni, quali Ivan Kušar e Rikard Katalinić Jeretov, ben presto hanno abbandonato questo settimanale a causa, molto probabilmente, delle discrepanze ideologiche e personali con lo Jakić. ${ }^{8}$ Un aspetto, quello della

6 Le nostre appendici, in Il Diritto Croato, Pola, 3 ottobre 1888, I, 1.

7 Un giudizio del Carducci sulla lingua croata, in Il Diritto Croato, Pola, 7 marzo 1894, VI, 23.

8 A tal proposito si veda G. B-g-č, Corrispondenza Aperta, in Il Diritto Croato, 7 marzo 1894, VI, 23. 
figura del traduttore, sicuramente basilare per comprendere a pieno la funzione di questo settimanale all'interno della storia letteraria istriana, e non solo, di fine Ottocento e che si auspica possa essere il soggetto di future analisi.

\section{Bibliografia}

Bassnett, Susan (2003). La traduzione. Teorie e pratica, Milano: Bompiani.

Bertoša, Miroslav/Matijašić, Robert [a cura di] (2005). Istarska Enciklopedija, Zagabria: Leksikografski Zavod Miroslav Krleža.

Buffoni, Franco (2007). Con il testo a fronte. Indagine sul tradurre e l'essere tradotti, Novara: Interlinea.

Cella, Sergio (1956). Giornalismo e stampa periodica in Istria, in: Atti e memorie della società istriana di archeologia e storia patria, IV, pp. 120-164.

Cronia, Arturo (1958). La conoscenza del mondo slavo in Italia, Venezia: Istituto di Studi Adriatici.

Damiani, Martina/Fioretti, Fabrizio (2017). I contenuti letterari nei periodici italiani dell'Istria (1846-1918) vol. I, Pola: Università Juraj Dobrila di Pola.

Eco, Umberto (2012). Dire quasi la stessa cosa. Esperienze di traduzione, Milano: Bompiani.

Fioretti, Fabrizio (2019). Ante Jakić e il settimanale «Il Diritto Croato» (Pola, 1888-1894), in: Studi sugli intellettuali europei tra Ottocento e Novecento [a cura di Martina Damiani/Fabrizio Fioretti], Pola: Università Juraj Dobrila di Pola, pp. 113-126.

Ivetic, Egidio [a cura di] (2006). Istria nel tempo, Rovigno: Centro di ricerche storiche di Rovigno.

Knez, Kristjan (2015). Lo studio del passato, le fonti e la valorizzazione del patrimonio culturale regionale: il ruolo della rivista La Provincia dell'Istria (1867-1894), in: Gli inizi della tutela dei beni culturali in Istria [a cura di Deborah Rogoznica], Capodistria: Histria Editiones, pp. 103-146.

Maier, Bruno (1996). La letteratura italiana dell'Istria dalle origini al Novecento, Trieste: Italo Svevo.

Nergaard, Siri [a cura di] (1993). La teoria della traduzione nella storia, Milano: Bompiani.

\section{Slučaj pulskog tjednika ll Diritto Croato [Hrvatsko pravo]}

ll Diritto Croato [Hrvatsko pravo] hrvatski je tjednik pisan na talijanskom jeziku koji se tiskao u Puli od 1888. do 1894. godine. Njegov je osnivač Antun ili Ante Jakić koji je stigao u Pulu kao svećenik, no ubrzo napušta svećenički poziv. Zbog svoga nemirnoga načina života i velikoga duga morao je prestati s izdavanjem tjednika i napustiti Pulu. Il Diritto Croato nastao je u veoma zahtjevno povijesno razdoblje Istre kada je zbog sukoba Talijana i Hrvata tisak postao pravo središte širenja kako političkih, tako i povijesno-kulturnih ideja. No dok je s talijanske strane tisak bio veoma razvijen, hrvatska je strana odgovorila 
tek Našom Slogom. Zbog toga, i kako bi mogao odgovoriti na stalne napade talijanske strane, Jakić osniva svoj tjednik kojem je bio cilj pokušati dokazati nadmoćnost hrvatske ali i istočne književnosti nad talijanskom kako bi Istranima približio ideje hrvatskog narodnog preporoda. Zato on u svom tjedniku prevodi stotine djela raznih hrvatskih autora koji jasno pokazuju kako prijevod nije samo puko prenošenje istoga teksta s jednoga koda u drugi, nego je i prenošenje emocija, ideala kao što je domoljublje te prenošenje hrvatske kulture i običaja.

Ključne riječi: Il Diritto Croato, Ante Jakić, Istra, XIX stoljeće, talijansko novinarstvo Istre. 
